\title{
Long Non-Coding RNA Duxap8 Facilitates Cell Proliferation and Induces Apoptosis in Colorectal Cancer via miR-5|9b/ZNF277 Axis
}

\author{
Hailiang Liang ${ }^{1} *$ \\ Jin Wang $\mathbb{B}^{2, *}$ \\ Peng Zhang ${ }^{1} *$ \\ Wei Yang ${ }^{3}$ \\ Yang Yang ${ }^{4}$ \\ Yin Zhi ${ }^{1}$ \\ Wei $\mathrm{Wu}^{3}$ \\ Xiaoqiang Dong (D)
}

'Department of General Surgery, The First Affiliated Hospital of Soochow University, Suzhou, 215006, People's Republic of China; ${ }^{2}$ Department of General Surgery, Suzhou Dushu Lake Hospital (Dushu Lake Hospital Affiliated to Soochow University), Suzhou, 215006, People's Republic of China; ${ }^{3}$ Department of General Surgery, The Affiliated Hospital of Yangzhou University, Yangzhou, 225000, People's Republic of China; ${ }^{4}$ Department of Anesthesiology, The Affiliated Hospital of Yangzhou University, Yangzhou, 225000, People's Republic of China

*These authors contributed equally to this work
Correspondence: Xiaoqiang Dong; Wei

Wu

Email dongxq@hotmail.com;

306834397@qq.com
Introduction: Long non-coding RNAs (LncRNAs) play a critical role in development and progression of various cancers. More and more researchers pay attention to the effect of lncRNA on regulating the cancer. However, the function and mechanism of Duxap 8 in colorectal cancer have not been studied.

Methods: Reverse transcription quantitative PCR (RT-qPCR), cell counting kit-8 (CCK-8), 5-ethynyl-20-deoxyuridine (EdU), colony formation assay, flow cytometry, TdT-mediated dUTP nick-end labeling (TUNEL), Western blot, hematoxylin-eosin staining (HE), in situ hybridization (ISH) analysis, immunohistochemistry (IHC) and tumor transplantation experiment were performed to investigate the function and mechanism of Duxap 8 in colorectal cancer.

Results: We found that the expression level of Duxap8 in colorectal cancer was closely correlated with tumor size $(\mathrm{P}=0.024)$, tumor depth $(\mathrm{P}=0.035)$ and lymphatic invasion $(\mathrm{P}$ $=0.067$ ) among 50 colorectal cancer patients. Then, we proved that the expression level of Duxap8 was significantly increased in human colorectal cancer tissues and cell lines. Functionally, Duxap 8 knockdown inhibited the proliferation and induced the apoptosis of colorectal cancer cells, while Duxap 8 overexpression facilitated the proliferation and suppressed the apoptosis in colorectal cancer in vitro. Moreover, knockdown of Duxap 8 inhibited the size and weight of tumors in mice injected with colorectal cancer cells, overexpression of Duxap8 promoted the growth of colorectal cancer cells in vivo. Mechanically, we found that Duxap 8 was principally located in the cytoplasm. Furthermore, Duxap 8 functioned as a competing endogenous RNA to induce the development and progression of colorectal cancer through sponging miR-519b-3p to upregulate ZNF277.

Discussion: Taken together, our results demonstrated that Duxap 8 enhanced the expression level of ZEB1 to promote via competing for miR-519b-3p, which might be a promising molecular therapeutic target of colorectal cancer.

Keywords: Duxap8, miR-519b-3p, ZNF277, colorectal cancer

\section{Introduction}

Colorectal cancer is the second most common cancer and the third leading cause of cancer-related deaths all over the world. ${ }^{1}$ Although technological advances in early detection and early intervention have improved the overall survival of colorectal cancer in recent years, the recurrence and metastasis rates of advanced colorectal cancer are high, and the prognosis remains poor. ${ }^{2,3}$ Moreover, the exact molecular mechanism underlying the development and progression of colorectal cancer is still unclear. Therefore, further molecular analysis of colorectal cancer is necessary to 
improve the understanding of the pathogenesis of colorectal cancer and develop new therapeutic strategies.

Long non-coding RANs (LncRNAs) are non-coding RNAs of at least 200 nucleotides in length that cannot encode proteins. Emerging evidences have indicated IncRNAs play a critical role in colorectal cancer and are associated with the treatment and prognosis of colorectal cancer. Damas et al report SNHG5 depletion induces cell cycle block and cell apoptosis in vitro and inhibits tumor in vivo, suggesting that Snhg5 acts as a potential molecular target for colorectal cancer. ${ }^{4}$ Han et al demonstrate that CRNDE knockdown suppresses cell proliferation and chemoresistance via regulating miR-181a-5p and Wnt/ $\beta$-catenin signaling. ${ }^{5}$ Ding et al prove that lncRNA H19/miR-29b-3p/ PGRN axis facilitates epithelial-mesenchymal transition of colorectal cancer via regulating the Wnt signaling. ${ }^{6}$ In addition, diverse lncRNAs oncogenous functions have also been studied in colorectal cancer, such as $\operatorname{Snh} g 1,{ }^{7} \mathrm{Ucal},{ }^{8} \mathrm{Uiclm},{ }^{9}$ Xist. ${ }^{10}$ Multiple lines of evidences expound that Duxap8 contributes to the tumorigenesis of numerous malignancies. ${ }^{11-13}$ However, the effects of long non-coding RNA Duxap 8 on development and progression of colorectal cancer have been rarely investigated.

In the present study, we found that high expression level of Duxap8 in colorectal cancer closely associated with tumor size $(\mathrm{P}=0.024)$, tumor depth $(\mathrm{P}=0.035)$ and lymphatic invasion $(\mathrm{P}=0.067)$ among 50 colorectal cancer patients. Moreover, we demonstrated that the expression level of Duxap 8 was significantly increased in tissues and cell lines of human colorectal cancer. Furthermore, Duxap8 knockdown inhibited cell proliferation and induced apoptosis in colorectal cancer in vitro as well as in vivo, while Duxap8 overexpression produced the opposite results. Mechanically, Duxap 8 served as a competing endogenous RNA of miR-519b-3p to elevate the expression level of ZNF277, which provided a novel insight into the treatment of colorectal cancer.

\section{Materials and Methods}

\section{Tissue Samples}

50 pairs of human colorectal cancer tissues and matched normal tissues were collected from our department The First Affiliated Hospital of Soochow University and all participants signed the written consent forms. To apply the clinical tissues for study, the approval from the Institutional Research Ethics Committee of the The First Affiliated Hospital of Soochow University (2021-024) were obtained. All patients provided informed consent according the Declaration of Helsinki. All clinical samples were stored at $-80^{\circ} \mathrm{C}$ until use.

\section{Cell Lines and Cell Culture}

The human colorectal cancer cell lines (HCT-8, Lovo, SW480, HT-29, H716) and normal cell line (NCM460) were purchased from the Cell Bank of the Chinese Academy of Science (Shanghai, China). Cells were maintained in high-glucose dulbecco's modified Eagle's Medium (DMEM) with 10\% fetal bovine serum (Gibco, NY, USA) and $100 \mathrm{U} / \mathrm{mL}$ penicillin/streptomycin (Corning, NY, USA) in a humidified incubator of $5 \% \mathrm{CO}_{2}$ atmosphere at $37^{\circ} \mathrm{C}$.

\section{Reverse Transcription Quantitative PCR (RT-qPCR) Analysis}

RNA was isolated by Trizol (Invitrogen, CA, USA) according to the manufacturer's protocols. RNAs were reverse transcribed by employing PrimeScript RT Master Mix (Takara, Nanjing, China) following the manufacturer's protocols. PCR amplification for Duxap8, miR519b-3p and ZNF277 was conducted with the SYBR Premix Ex TaqTM Kit (Takara, Nanjing, China). GAPDH and U6 were used to normalize the expression of Duxap8, miR-519b-3p and ZNF277.

\section{Cell Proliferation Assay}

A Cell Counting Kit-8 (CCK-8, Beyotime Institute of Biotechnology, China) and a 5-ethynyl-20-deoxyuridine (EdU, Ribobio, Shanghai, China) assay kit were adopted to examine nasopharyngeal cancer cell proliferation following the manufacturer's instructions. Cell viability and EdU positive cells were presented as the percentage. Colony formation assay was also performed to detect cell proliferation. The experimental steps were as follows. The cells (400 cells per well) were uniformly placed in a 6-well plate for 14 days. The cells were flushed twice with PBS, and fixed with methanol for $15 \mathrm{~min}$, and dyed with $0.5 \%$ crystal violet for $15 \mathrm{~min}$ at room temperature. After washing off the dye, colonies were photographed and quantified.

\section{Cell Apoptosis Assay}

Flow cytometry was conducted to measure colorectal cancer cell apoptosis. Cells $\left(1 \times 10^{6}\right.$ cells/well $)$ were seeded in sixwell plates. The cells were harvested at $24 \mathrm{~h}$ after treatment and therewith apoptotic cells were detected through a FACScalibur Flow Cytometry (BD Biosciences, CA, 
USA) by annexin-V fluorescein isothiocyanate (FITC) and propidium iodide (PI) apoptosis detection kit (Invitrogen). TUNEL assay (Roche, Mannheim, Germany) was also conducted to detect cell apoptosis. These assays were carried out according to the manufacturer's protocols.

\section{Dual Luciferase Reporter Assay}

Bioinformatics tools TargetScanHuman 7.1 were employed to predict the Duxap8 binding sites of miR519b-3p and the miR-519b-3p binding sites of ZNF277. Human colorectal cancer cells were transfected with 150 ng of empty pmirGLO-NC, pmirGLO-Duxap8-WT or pmirGLO-Duxap8-Mut (GenePharma) and the miR-519b$3 p$ mimics or mimics NC were co-transfected into cells by using Lipofectamine 2000 (Invitrogen) following the manufacturer's procedures. The relative luciferase activity was measured after transfection. To verify the targeting relationship between miR-519b-3p and ZNF277, the principle is the same as above.

\section{Western Blot Analysis}

Total proteins in tissues or cells were extracted with RIPA lysate buffer (Beyotime Inc., China) including benzosulfonyl fluoride (Beyotime Inc., China) and protease inhibitor cocktail (Beyotime Inc., China). SDS-PAGE was applied to isolate equal amounts of protein and then samples were transferred to PVDF membrane (Millipore Inc., USA). The membrane was incubated overnight with the primary antibody at $4^{\circ} \mathrm{C}$ and then incubated with HRP coupled secondary antibody (Santa Cruz Inc., China). Anti-GAPDH antibody (Santa Cruz Inc., China) was utilized as an internal control. Protein expression levels were measured using ECL substrate (Beyotime Inc., China).

\section{RNA Immunoprecipitation (RIP)}

The RIP assay was implemented with a Magna RIPTM RNA-Binding Protein Immunoprecipitation Kit (Millipore) in line with the manufacturer's recommendations. AGO2 antibody was applied for RIP (Cell Signaling Technology, USA) with IgG antibody (Cell Signaling Technology) as the negative control. RT-qPCR analysis was used to determine the abundance of RNAs specifically binding to $\mathrm{AGO} 2$.

\section{Subcellular Fractionation Analysis}

Subcellular fractionation analysis was performed according to a previously described method. ${ }^{14}$

\section{Hematoxylin-Eosin Staining (HE) and Immunohistochemical (IHC) Assays}

For HE assay, the slices of tumor tissues were stained with hematoxylin for $5 \mathrm{~min}$, then washed for $1 \mathrm{~min}$, and restored to blue by $1 \%$ ammonia ( $30 \mathrm{~s})$. Then, slices were swashed with running water $(1 \mathrm{~min})$. After, slices were stained by $0.5 \% \mathrm{HE}$ or $1 \mathrm{~min}$, flushed for $30 \mathrm{~s}$ then transparentized and finally mounted with neutral gum. For IHC assay, the appropriate antibody diluent and antigen repair treatment methods were selected following the manufacturer's instructions. The paraffin-embedded tissue was dewaxed in xylene, dehydrated in ethanol, and $3 \% \mathrm{H}_{2} \mathrm{O}_{2}$ blocked for $10 \mathrm{~min}$. The antigen was extracted in $95^{\circ} \mathrm{C}$ EDTA buffer for $15-20 \mathrm{~min}$. Sections were incubated with Ki67 antibody (Santa Cruz, USA) overnight at $4^{\circ} \mathrm{C}$. After washing 3 times with PBS, sections were incubated with the secondary antibody coupled to HRP (Santa Cruz Inc., USA) at $37^{\circ} \mathrm{C}$ for $50 \mathrm{~min}$. Finally, DAB chromogen (Beyotime Inc., China) was added, and then slices were washed with water and stained with hematoxylin.

\section{Tumor Transplantation Experiment}

All procedures involving mice in this research were approved by Institutional Research Committee of The First Affiliated Hospital of Soochow University (2021YKL03-K009). All animals were handled following to the Standards for Laboratory Animals (GB14925-2001) and the Guideline on the Humane Treatment of Laboratory Animals (MOST 2006a) established by the PR China. All nude mice aged 6-7 weeks and weighing 22-24 g (Shanghai Experimental Animal Centre) were used. Tumors were occurred by subcutaneously injection into the right flank of nude mice with $1 \times 10^{6}$ stably transfected SW480 cells transfected with Sh-NC, ShDuxap8, Lv-NC and Lv-DUXP8. Mice weight and tumor volumes were measured weekly over a period of 4 weeks.

\section{Statistical Analyses}

All experiments were repeated three times independently. All data were presented as the mean \pm SD of 3 independent experiments. Difference between two or multiple groups was analyzed by $t$-test or one-way ANOVA using SPSS 17.0. Statistical significance was set at $P<0.05$. 


\section{Results}

Up-Regulation of DUXAP8 Predicts the Poor Clinicopathological Characteristics of Colorectal Cancer Patients

To analyze the association between Duxap 8 expression level and clinicopathologic characteristics, the 50 patients with colorectal cancer were classified into high Duxap8 expression group and low Duxap 8 expression group using the median expression level of Duxap 8 in colorectal cancer tissues. Obviously, high expression level of Duxap8 in colorectal cancer was positively correlated with tumor size $(P=0.024)$, tumor depth $(P=0.035)$ and lymphatic invasion $(P=0.067)$ (Table 1$)$.

\section{DUXAP8 is Increased in Colorectal}

\section{Cancer}

Based on the above findings, we intended to explore the expression pattern of Duxap8 in colorectal cancer. To determine the expression level of Duxap 8 in human tissues and cell lines, RT-qPCR was conducted. The results showed that the expression level of Duxap8 was significantly up-regulated in colorectal cancer tissues, when compared with that in adjacent non-cancer tissues (Figure 1A). Then, we found that the expression level of Duxap 8 was prominently increased in III and IV stages

Table I Upregulation of Duxap8 Predicts the Poor Clinicopathological Characteristics of Colorectal Cancer Patients

\begin{tabular}{|c|c|c|c|}
\hline \multirow{2}{*}{$\begin{array}{l}\text { Clinical } \\
\text { Parameters }\end{array}$} & \multicolumn{2}{|c|}{ DUXAP8 } & \multirow[t]{2}{*}{$P$ value } \\
\hline & $\begin{array}{c}\text { Low } \\
\text { Expression } \\
(n=21)\end{array}$ & $\begin{array}{c}\text { High } \\
\text { Expression } \\
(n=29)\end{array}$ & \\
\hline Age & & & 0.103 \\
\hline$>60$ & 8 & 17 & \\
\hline$\leq 60$ & 13 & 12 & \\
\hline FIGO & & & 0.035 \\
\hline$|\sim| \mid$ & 10 & 9 & \\
\hline III IV & 11 & 20 & \\
\hline Tumor Size & & & 0.024 \\
\hline$>2 \mathrm{~cm}$ & 9 & 18 & \\
\hline$\leq 2 \mathrm{~cm}$ & 12 & 11 & \\
\hline Lymph nodes & & & 0.067 \\
\hline status & & & \\
\hline Negative & 14 & 19 & \\
\hline Positve & 7 & 10 & \\
\hline
\end{tabular}

of colorectal cancer compared with that in I and II stages of colorectal cancer (Figure 1B). Consistently, RT-qPCR analysis indicated that the expression level of Duxap8 was higher in human colorectal cancer cell lines (HCT-8, Lovo, SW480, HT-29, H716) than human normal colon epithelial cell-line NCM460 (Figure 1C). All these data indicated a positive relationship between Duxap 8 and the colorectal cancer.

\section{DUXAP8 Contributes to Cell Proliferation of Colorectal Cancer in vitro}

To examine the effects of Duxap 8 on regulating colorectal cancer, we first investigated the function of Duxap 8 in cell proliferation of colorectal cancer. The efficiency of Duxap8 knockdown or overexpression was examined in SW480 and HT-29 colorectal cancer cells by RT-qPCR. The results indicated that the expression level of Duxap 8 was observably decreased in Sh-Duxap 8 group compared with that in Sh-NC group, while the expression level of Duxap8 was greatly increased in Lv-Duxap8 group compared with that in Lv-NC group (Figure 2A). Then, CCK-8 assay showed that the proliferation capability of SW480 and HT-29 cells was significantly inhibited in Sh-Duxap8 group compared with that in Sh-NC group, while overexpression of Duxap8 promoted colorectal cancer cell proliferation (Figure 2B). Moreover, EdU and colony formation assays further validated the activation role of Duxap8 in the proliferation capability of SW480 and HT29 cells (Figure 2C and D). Consistently, Western blot analysis illustrated that depletion of Duxap8 overtly decreased the protein expression levels of Ki67 and PCNA in SW480 and HT-29 cells, whereas the protein levels of Ki67 and PCNA were significantly increased in SW480 and HT-29 cells in Lv-Duxap8 group compared with that in Lv-NC group (Figure 2E). All these data suggested that Duxap 8 knockdown inhibited cell proliferation of colorectal cancer, while Duxap 8 overexpression promoted colorectal cancer cell growth.

\section{DUXAP8 Inhibits Cell Apoptosis of Colorectal Cancer in vitro}

Secondly, we investigated the effects of Duxap 8 on the apoptosis of colorectal cancer. Flow cytometry assay showed that knockdown of Duxap 8 greatly increased the apoptosis rate in SW480 and HT-29 cells, while overexpression of Duxap8 led to the opposite results (Figure 3A). 
A

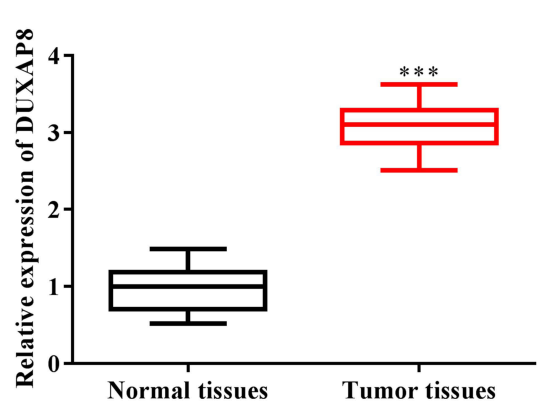

C

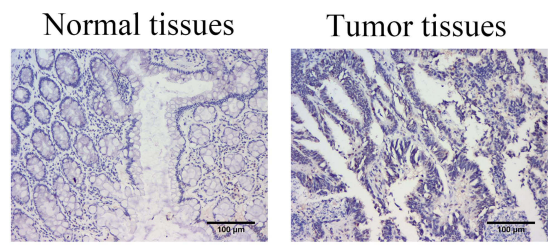

B

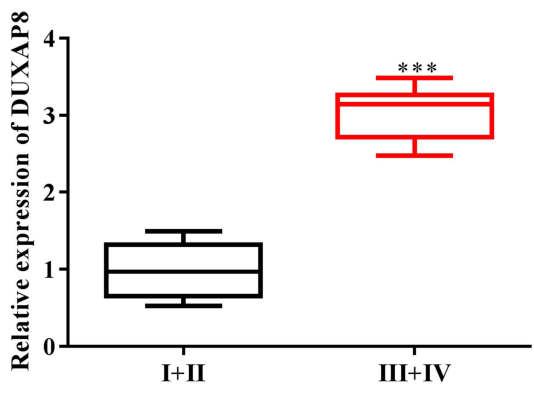

D

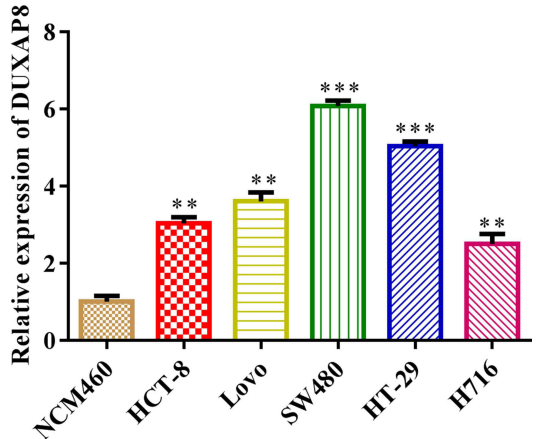

Figure I DUXAP8 is increased in colorectal cancer. (A) RT-qPCR analysis of the expression level of Duxap8 in colorectal cancer tissues and adjacent non-cancer tissues. $* * * P<0.001$ vs normal tissues group. (B) RT-qPCR analysis of the expression level of Duxap8 in different stages of colorectal cancer. $* * * P<0.00$ I vs I + II group. (C) Subcellular fractionation analysis of the location of Duxap8. (D) RT-qPCR analysis of the expression level of Duxap8 in colorectal cancer cell lines. **P $<0.0 \mathrm{I}$, ***P $<0.00 \mathrm{I}$ vs NCM460 group. All results were presented as the mean \pm SD from at least three separate experiments.

Moreover, TUNEL assay also confirmed the inhibitory effects of Duxap8 on colorectal cancer cell apoptosis (Figure 3B). Similarly, we found that inhibition of Duxap 8 remarkably decreased Bcl-2 level and heightened the expression levels of apoptosis-related proteins, including Bax, Cleaved-caspase-3 and Cleaved-caspase-9, while Bcl-2 level was increased and the expression levels of Bax, Cleaved-caspase-3 and Cleaved-caspase-9 were significantly downregulated owing to enhanced expression of Duxap8 (Figure 3C). All these data suggested that Duxap8 blocked cell apoptosis of colorectal cancer.

\section{DUXAP8 Facilitates Cell Growth of Colorectal Cancer in vivo}

Subsequently, we identified the effects of Duxap 8 on colorectal cancer in vivo by the assay of transplanted tumor in nude mice. Tumor phenotypes are presented in Figure 4A. We found that the tumor volume was significantly decreased by suppression of DXUAP8, while the tumor size was larger in Lv-Duxap8 group than Lv-NC (Figure 4B). Then, HE assay showed the same effects of DXUAP8 on tumor growth (Figure 4C).
Subsequently, the tumor weight was determined in (Figure 4D). The results showed that the tumor weight was significantly decreased in Sh-DXUAP8 group compared with that in Sh-NC, while forced expression of DXUAP8 increased the weight of xenografts (Figure 4D). Similarly, IHC assay indicated that knockdown of DXUAP8 resulted in the reduction of Ki67 level and the increased expression level of Cleaved-caspase-3, the opposite consequences were obtained when DXUAP8 was overexpressed (Figure 4D). All these results suggested that Duxap 8 promoted the growth of colorectal cancer in vivo.

\section{DUXAP8 Negatively Regulates miR-5I9b-3p}

As Duxap8 was mainly expressed in the cytoplasm of colorectal cancer cells, we presumed that Duxap 8 might exert its function by serving as a competing endogenous RNA (ceRNA). The potential targets of Duxap 8 were predicted by the bioinformatics databases. The miR-519b-3p contained a putative binding site, which is a complementary sequence of Duxap8 (Figure 5A). Dual luciferase reporter assay 

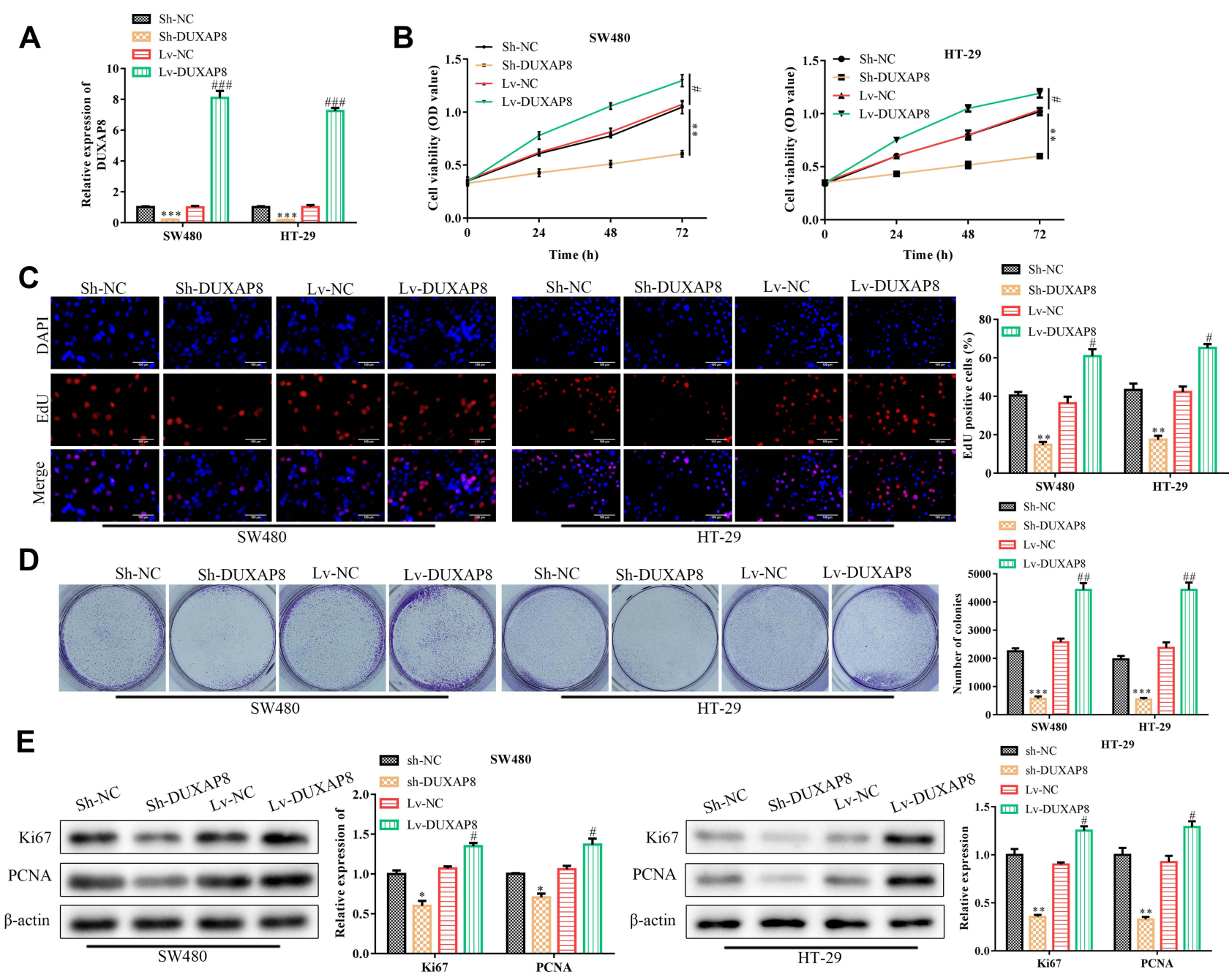

sh-NC

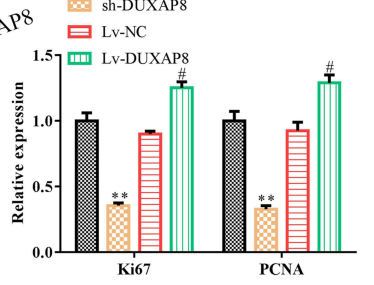

Figure 2 Duxap8 contributes to cell proliferation of colorectal cancer in vitro. (A) RT-qPCR analysis of the efficiency of Duxap8 knockdown or overexpression. (B) CCK-8 analysis of the proliferation of SW480 and HT-29 colorectal cancer cells. (C) EdU assay analysis of the proliferation of SW480 and HT-29 colorectal cancer cells. (D) Colony formation assay analysis of the proliferation of SW480 and HT-29 colorectal cancer cells. (E) Western blot analysis of the proliferation-related protein expression levels in

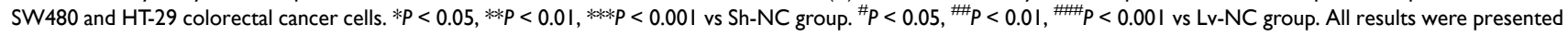
as the mean \pm SD from at least three separate experiments.

indicated that only the luciferase activity of Duxap8-WT was impaired by miR-519-3p mimics and no significant difference was observed in mutant forms of Duxap8 (Figure 5B). RIP experiment further confirmed that Duxap 8 directly combined with miR-519b-3p (Figure 5C). In addition, RT-qPCR assay showed that the expression level of miR-519b-3p was significantly decreased in human colorectal cancer tissues compared with that in human adjacent non-cancer tissues (Figure 5D). Meanwhile, miR-519b-3p was weakly expressed in human colorectal cancer cell lines (HCT-8, Lovo, SW480, HT-29, H716) compared with that in human normal colon epithelial cell NCM460 (Figure 5E). Furthermore, we found that miR-519b-3p level was elevated by suppression of Duxap 8 and inversely the diminution of miR-519b-3p expression level occurred due to Duxap8 overexpression (Figure 5F). All these data demonstrated that Duxap8 negatively regulated miR-519b-3p.

\section{miR-519b-3p Targets ZNF277 in Colorectal Cancer}

Thereafter, we carried out bioinformatics analysis and discovered that ZNF277 possessed the predicted binding sites with miR-519b-3p (Figure 6A). Dual luciferase reporter assay miR-519-3p mimics only lessened the luciferase activity of ZNF277-WT, suggesting that miR-519b-3p directly bound to ZNF277 (Figure 6B). In addition, RT-qPCR delineated that the expression level of ZNF277 was significantly increased in human colorectal cancer tissues in contrast with that in human adjacent non-cancer tissues (Figure 6C). Likewise, ZNF277 level was higher in colorectal cancer 


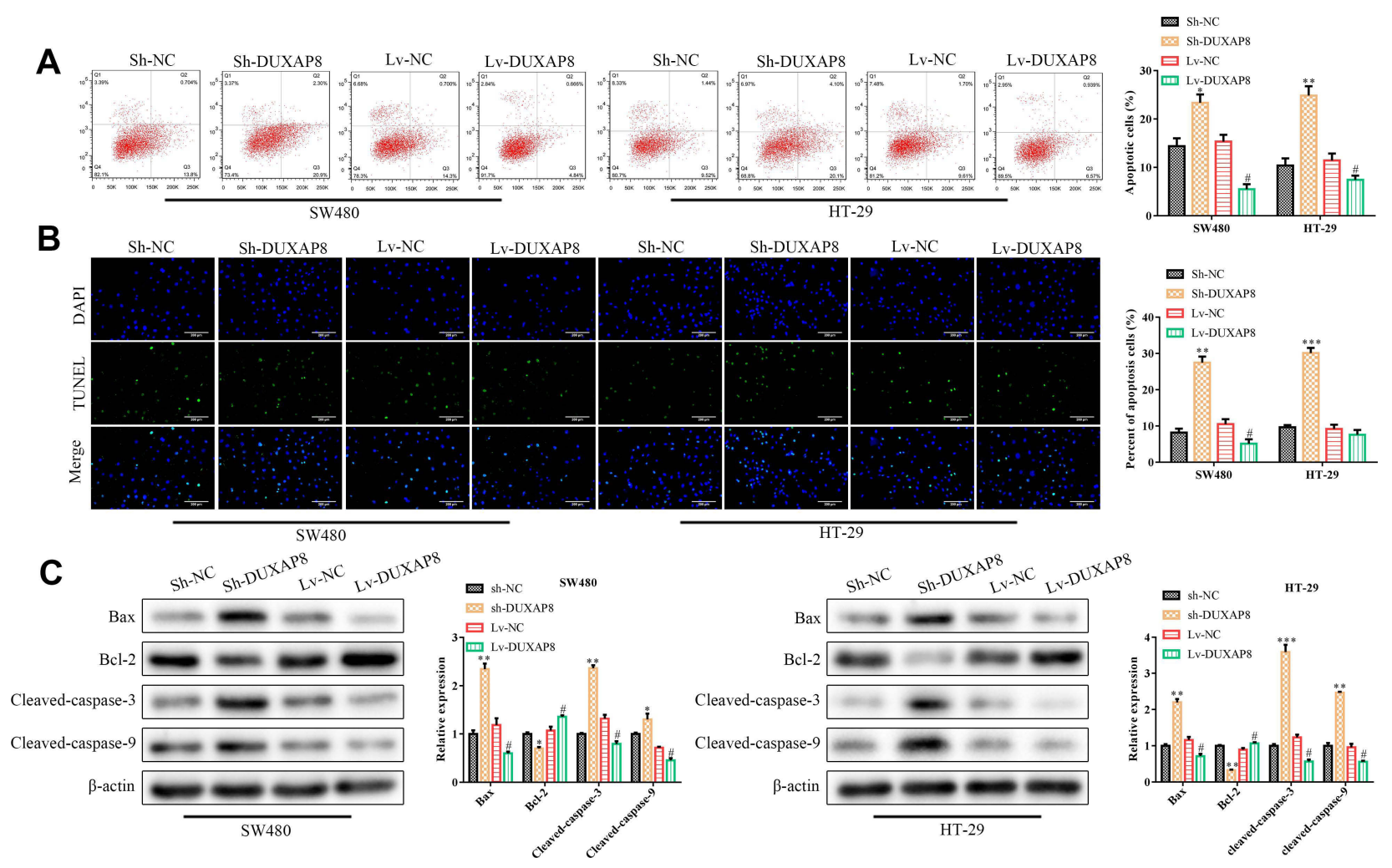

Figure 3 Duxap8 inhibits cell apoptosis of colorectal cancer in vitro. (A) Flow cytometry analysis of the apoptosis of SW480 and HT-29 colorectal cancer cells. (B) TUNEL analysis of the apoptosis of SW480 and HT-29 colorectal cancer cells. (C) Western blot analysis of the apoptosis-related protein expression levels in SW480 and HT-29 colorectal cancer cells. ${ }^{* P}<0.05,{ }^{* * P}<0.01$, ${ }^{* * * P}<0.00$ I vs Sh-NC group. ${ }^{\#} P<0.05$ vs Lv-NC group. All results were presented as the mean \pm SD from at least three separate experiments.

cells than normal cells (Figure 6D). Furthermore, we found that the expression level of ZNF277 was dramatically declined in SW480 and HT-29 cells in miR-519b-3p mimics group compared with that in mimics NC group, while inhibition of miR-519b-3p fortified ZNF277 level (Figure 6E). Based on the foregoing results, we concluded that miR519b-3p targeted ZNF277. In order to verify the CeRNAs mechanism, the RIP assays was testified for illustrating the direct binding function as shown in Figure 6F And confirmed that DUXAP8 could interact with ZNF277.

\section{Regulation of ZNF277 by DUXAP8 is Mediated by miR-519b-3p}

To investigate whether regulation of ZNF277 by Duxap8 was mediated by miR-519b-3p, we implemented rescue experiments. RT-qPCR was performed to verify the expression level of ZNF277 in SW480 and HT-29 colorectal cancer cells after transfection. Our observations disclosed that the decreased expression level of ZNF277 caused by Duxap8 depletion was increased by miR-519b-3p inhibitors and then retrieved by silencing of ZNF277 (Figure 7A). The results of
Western blot showed that ZNF277 expression at protein level exhibited the same trend (Figure 7B). Then, CCK-8 and EdU assays illuminated that the proliferation of SW480 and HT29 colorectal cancer cells inhibited by Duxap8 downregulation was promoted by inhibition of miR-519b-3p and recovered by depletion of ZNF277 (Figure 7C and D). In agreement with these findings, we uncovered that miR-519b$3 p$ increased the declined levels of Ki67 and PCNA induced by Duxap 8 knockdown, the restoration of Ki67 and PCNA expression levels occurred when ZNF277 was silenced (Figure 7E). The results of flow cytometry analysis and TUNEL assay indicated that the apoptosis of SW480 and HT-29 colorectal cancer cells promoted by repression of Duxap 8 was reversed by miR-519b-3p inhibitors and subsequently depletion of ZNF277 abolished the effects of miR$519 b-3 p$ inhibitors on cell apoptosis (Figure 7F and G). Consistently, Western blot further confirmed that the role of Duxap8/miR-519b-3p/ZNF277 in the apoptosis of SW480 and HT-29 colorectal cancer cells (Figure 7H). All these data demonstrated that regulation of ZNF277 by Duxap8 was mediated by miR-519b-3p. 
A
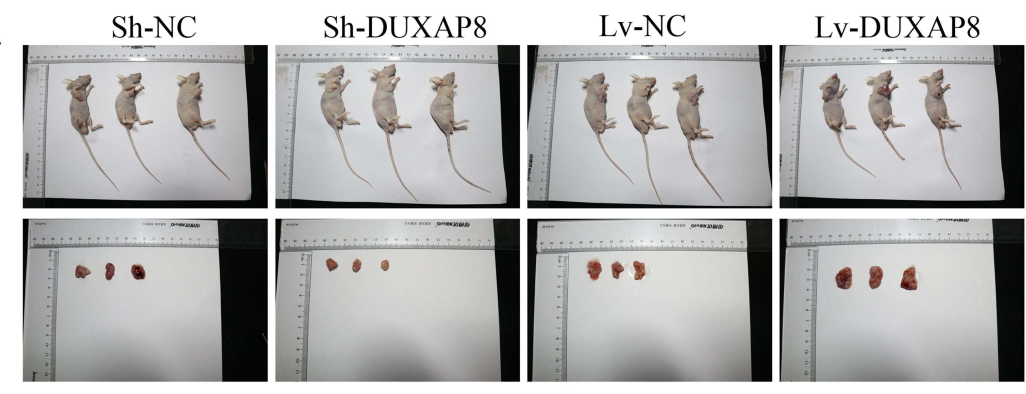

C

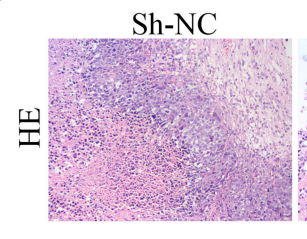

E

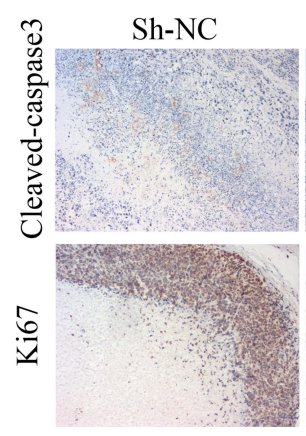

Sh-DUXAP8

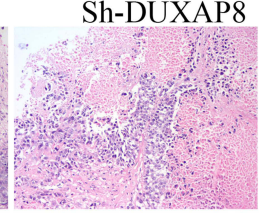

Sh-DUXAP8
$\mathrm{Lv}-\mathrm{NC}$
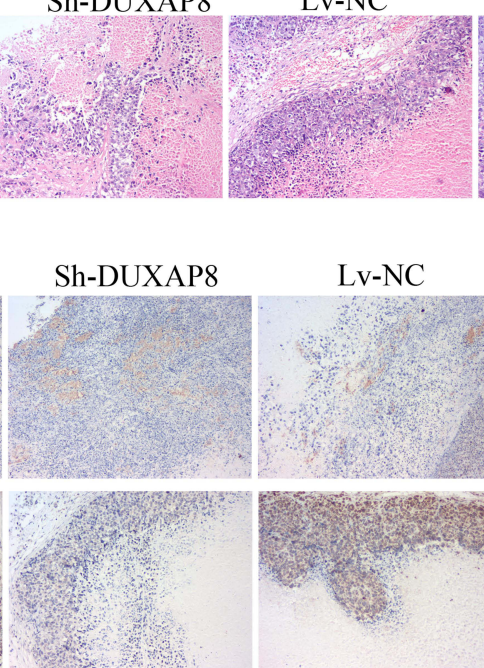

$\mathrm{Lv}-\mathrm{NC}$

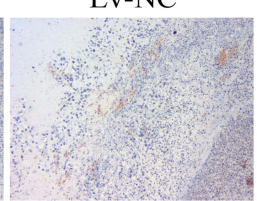

Lv-DUXAP8

Lv-DUXAP8

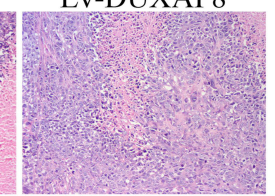

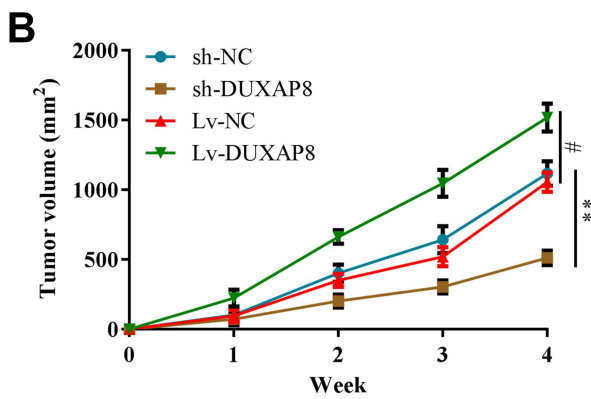

D

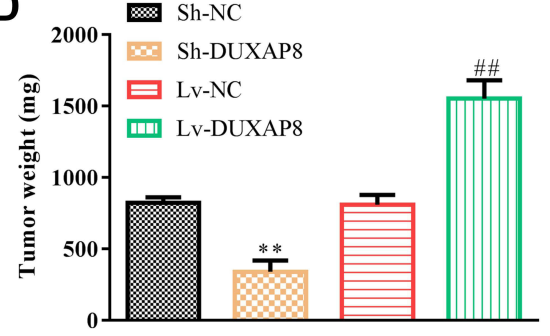

Figure 4 Duxap8 facilitates cell growth of colorectal cancer in vivo. (A) Images of xenografts were presented. (B) The growth curve of tumors. (C) HE analysis of colorectal cancer tissues. (D) Tumor weights. (E) IHC analysis of Ki67 and Cleaved-caspase-3. ${ }^{* * P}<0.0 \mathrm{I}$ vs Sh-NC group. ${ }^{\#} P<0.05$, ${ }^{\#} P<0.0 \mathrm{I}$ vs Lv-NC group.

\section{Discussion}

In our study, we found that high expression level of Duxap8 in colorectal cancer was significantly correlated with tumor size, tumor depth and lymphatic invasion through clinical data analysis. Moreover, we demonstrated that the expression level of Duxap8 was greatly upregulated in human colorectal cancer tissues and cell lines. Moreover, knockdown of Duxap8 repressed cell proliferation and promoted cell apoptosis in colorectal cancer in vitro, while overexpression of Duxap 8 caused the opposite results in vitro. Furthermore, Duxap 8 facilitated colorectal cancer tumor growth in vivo. Mechanically, Duxap8 functioned as an oncogene via sponging miR-519b-3p to regulating the expression level of ZNF277.

LncRNAs are increasingly important as molecular targets for cancer treatment. Sun et al have reported that knockdown of Duxap8 inhibits non-small-cell lung cancer cell growth, migration and invasion and induced apoptosis both in vitro and in vivo, which acts as a potential molecular therapeutic target for non-small-cell lung cancer. ${ }^{11}$ Ma et al have demonstrated that Duxap 8 promotes cell proliferation and migration of gastric cancer by epigenetically silencing PLEKHO1 expression, suggesting Duxap8 as a promising therapeutic target for gastric cancer. ${ }^{12}$ According to the statistics of their studies, we found that the expression level of Duxap 8 was abnormally expressed in multiple malignancies. Notably, our results showed that the expression level of Duxap 8 was significantly increased by $10-15$ times in colorectal cancer tissues and cell lines, indicating that Duxap8 was a sensitive lncRNA in colorectal cancer and was closely associated with the development and progression of the tumor.

Emerging evidences have indicated that Duxap8 acts as an oncogene to regulate the development and progression of various cancers. Lian et al prove that Duxap 8 facilitates cell proliferation and inhibits cell apoptosis of pancreatic cancer, 
A
B
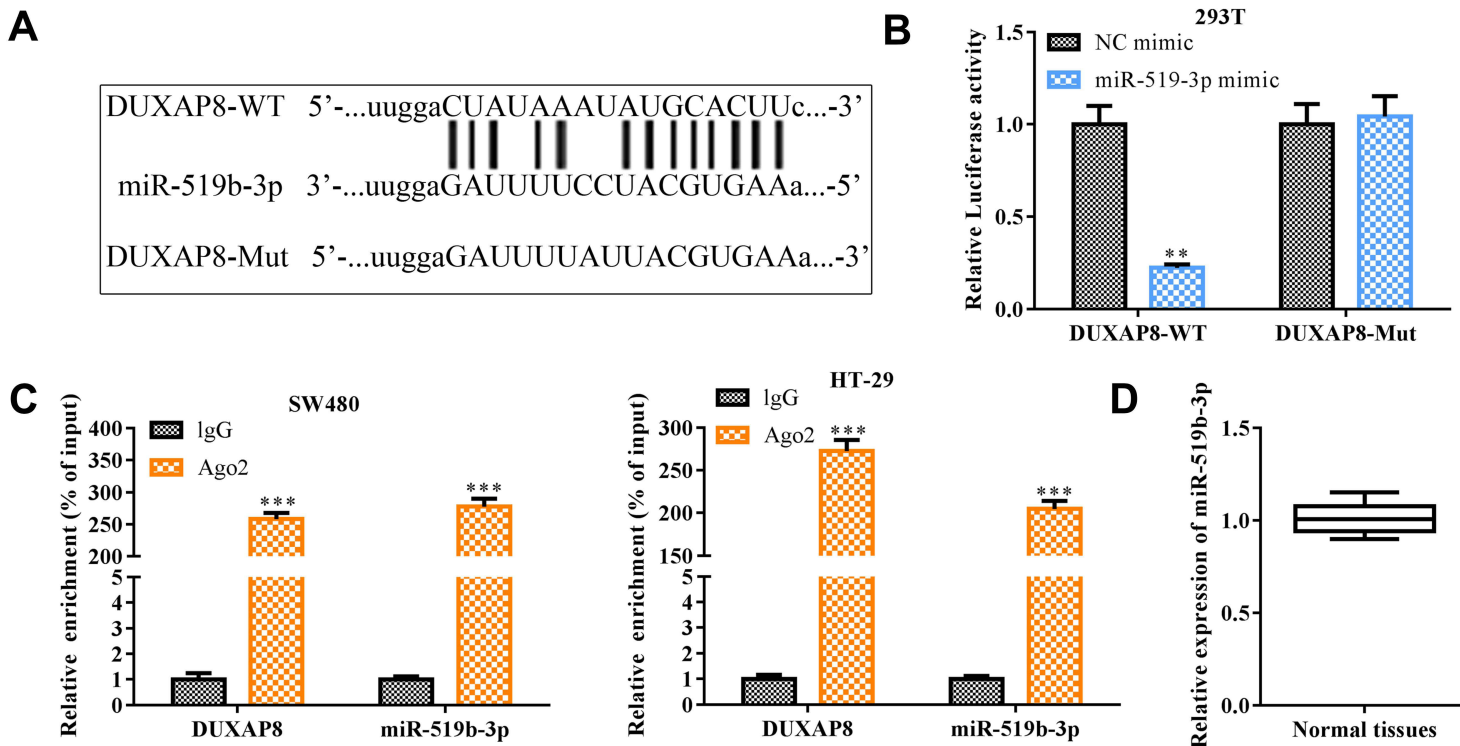

E

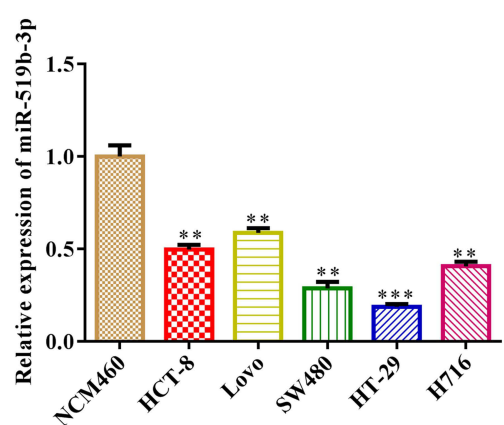

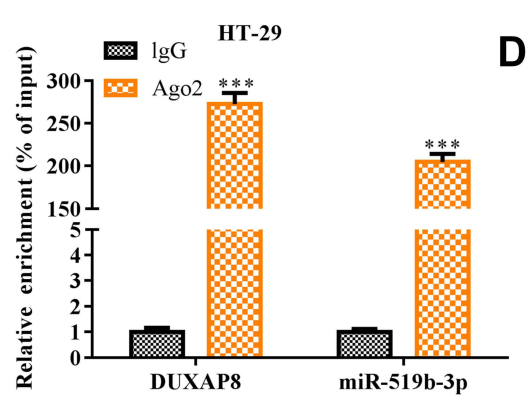

$\mathbf{F}$

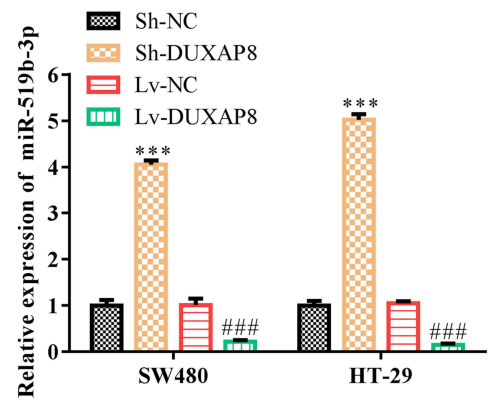

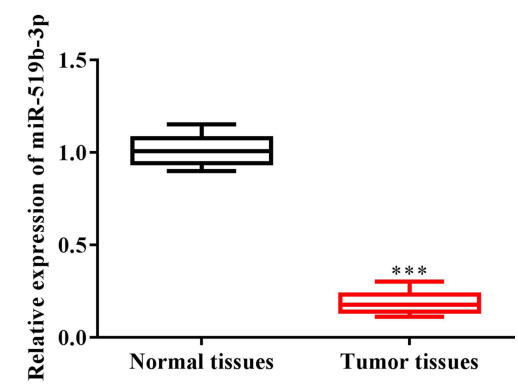

Figure 5 Duxap8 negatively regulates miR-5I9b-3p. (A) Conservation of Duxap8 in the binding site of miR-5I9b-3p was predicted. (B) Dual-luciferase reporter assay. (C) RIP experiment. (D) RT-qPCR analysis of miR-519b-3p in colorectal cancer tissues and adjacent non-cancer tissues. (E) RT-qPCR analysis of the expression level of miR$519 b-3 p$ in colorectal cancer cell lines. (F) RT-qPCR analyses of the expression level of miR-5I9b-3p in SW480 and HT-29 colorectal cancer cells. $* * * P<0.00$ I vs normal tissues group. ${ }^{* * P}<0.01$, ${ }^{* * *} P<0.00 \mathrm{I}$ vs Sh-NC group. ${ }^{\#} P<0.001$ vs Lv-NC group. All results were presented as the mean \pm SD from at least three separate experiments.

which might represent a promising biomarker of pancreatic cancer. $^{13}$ Lin et al point out that Duxap8 promotes cell proliferation of bladder cancer via regulating the expression level of PTEN. ${ }^{15}$ In addition, Duxap 8 was related with the development and progression of esophageal squamous cell cancer, ${ }^{16}$ renal cell cancer, ${ }^{17}$ and hepatocellular cancer. ${ }^{18}$ All these investigations provide strong evidences that Duxap 8 is participated in the tumorigenesis of human cancer through affecting cell proliferation, apoptosis, migration and invasion. Our studies showed that Duxap 8 accelerated colorectal cancer by modulating the proliferation and apoptosis. These findings were consistent with the results of previous studies. More importantly, our results further suggested the effects of Duxap8 on migration and invasion.

A myriad of literatures justify that lncRNAs can serve as endogenous molecular sponges to compete for miRNAs so that to negatively regulate miRNA expression. ${ }^{19}$ Since the subcellular fractionation analysis revealed the main expression of Duxap 8 in the cytoplasm, we hypothesized that Duxap 8 executed its function via a ceRNA network. Through employment of bioinformatics databases, we found that Duxap 8 harbored miR-519b-3p binding sites. The inhibitory effects of miR$519 b-3 p$ on cancer have been proven, especially in colorectal cancer. For instance, Luo et al have reported that miR-519b-3p facilitates responsiveness of preoperative chemoradiotherapy in rectal cancer. ${ }^{20}$ Shen et al have demonstrated that miR-519b$3 p$ blocks the cell cycle of Hep-2 cells in the G2/M phase. ${ }^{21}$ miR-519b-3p inhibits the proliferation and invasion in colorectal cancer via modulating the uMtCK/Wnt signaling pathway. $^{22}$ As a result, a targeting relationship between Duxap8 and miR-519b-3p was worth noted. We testified that Duxap 8 worked as a sponge of miR-519b-3p. Besides, further 
A

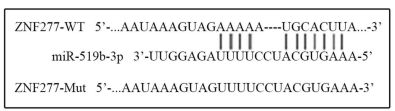

D

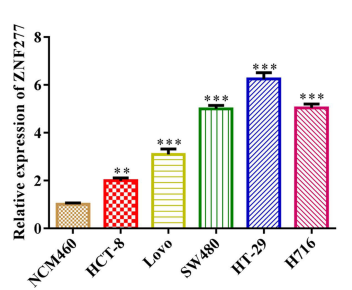

B

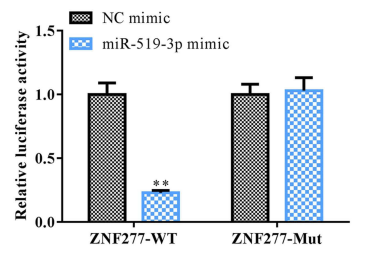

E

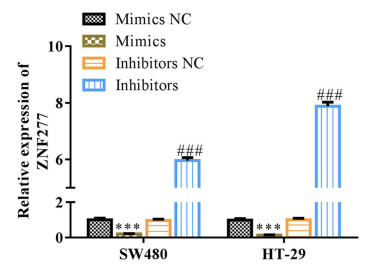

C

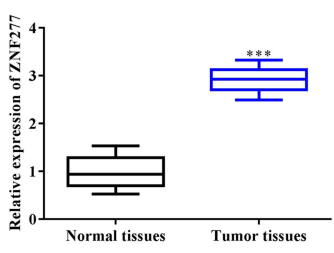

F

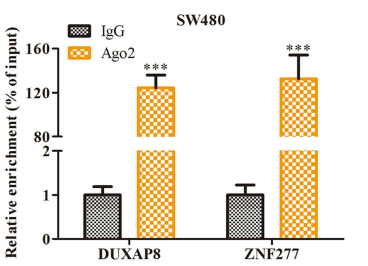

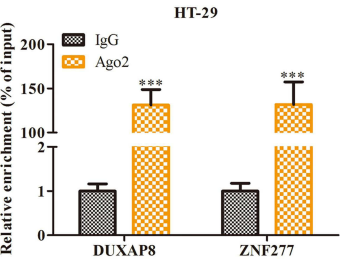

Figure 6 miR-519b-3p targets ZNF277 in colorectal cancer. (A) Conservation of miR-519b-3p in the binding site of ZNF277 was predicted. (B) Relative luciferase activity was performed by dual-luciferase reporter assay. $* * P<0.01$ vs ZNF277 WT + Mimics NC group. (C) RT-qPCR analysis of ZNF277 expression level in colorectal cancer tissues and adjacent non-cancer tissues. $* * * P<0.001$ vs normal tissues group. (D) RT-qPCR analysis of the expression level of ZNF277 in colorectal cancer cell lines. $* * P<$ 0.01 , ***P < 0.001 vs NCM460 group. (E) RT-qPCR analysis of the expression level of ZNF277 in SW480 and HT-29 colorectal cancer cells. ***P < $0.00 \mathrm{I}$ vs Mimics NC group. ${ }^{\# \#} P<0.00$ I vs Inhibitors NC group. (F) The RIP assays was testified for illustrating the direct binding function of DUXAP8 and ZNF277. $* * * P<0.00$ I vs Ig G group. All results were presented as the mean \pm SD from at least three separate experiments.

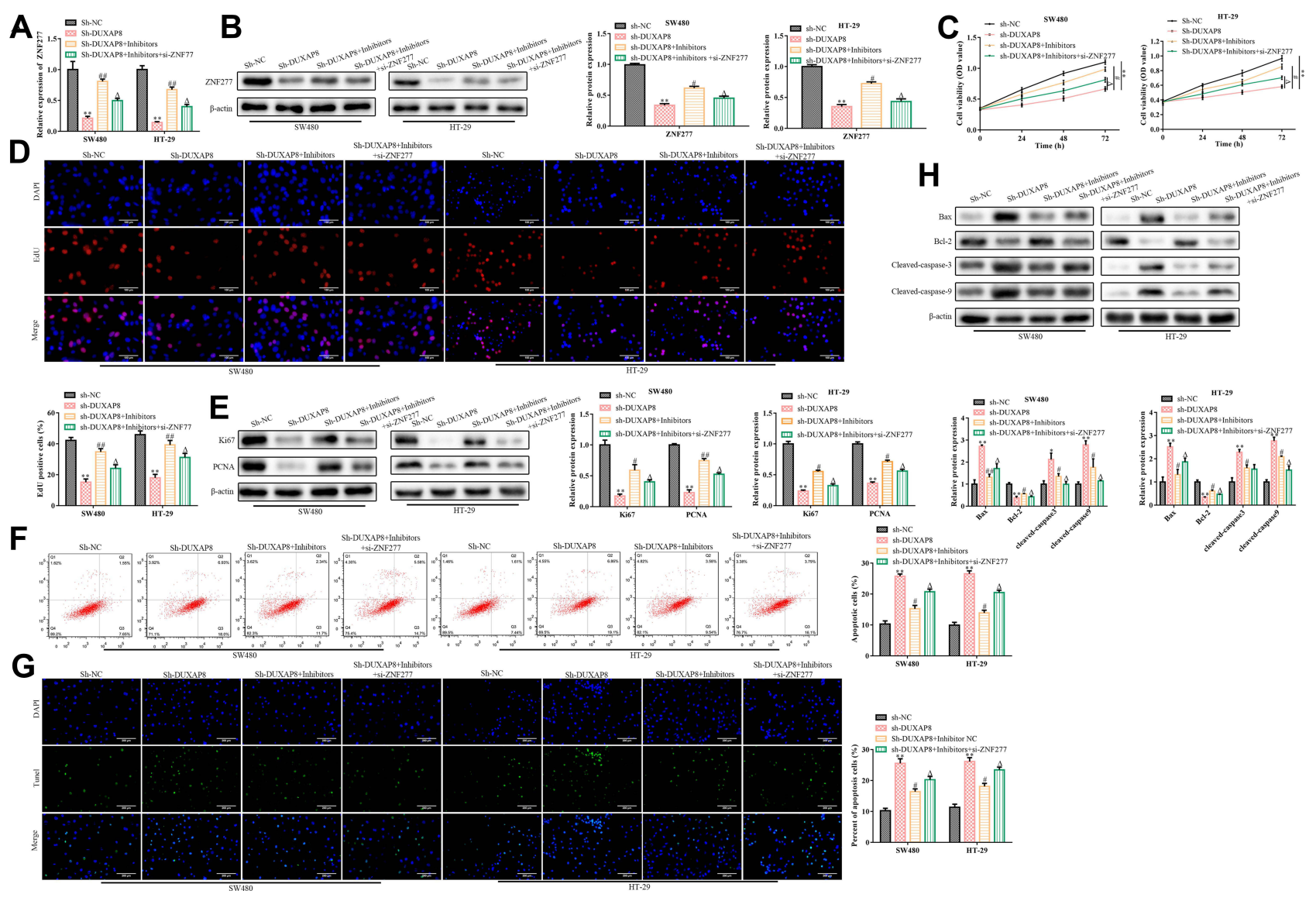

Figure 7 Regulation of ZNF277 by Duxap8 is mediated by miR-519b-3p. (A) RT-qPCR analysis of the expression level of ZNF277 in SW480 and HT-29 colorectal cancer cells. (B) Western blot analysis of the expression level of ZNF277 in SW480 and HT-29 colorectal cancer cells. (C) CCK-8 assay. (D) EdU assay. (E) Protein expression levels of key regulators involved in cell proliferation. (F) Flow cytometry analysis. (G) TUNEL assay. (H) Western blot analysis of expression levels of BAX, BCL-2, cleavedCaspase 3, and cleaved-Caspase 9. ${ }^{* * P} p<0.01$ vs Sh-NC group. ${ }^{\#} p<0.05,{ }^{\#} p<0.001$ vs Sh-Duxap8 group. ${ }^{\Delta} p<0.05$ vs Sh-Duxap8 + Inhibitors NC group. All results were presented as the mean \pm SD from at least three separate experiments. 
assays indicated that Duxap 8 enhanced the expression level of oncogene ZNF277 to induce colorectal cancer development by miR-519b-3p-dependent mechanism.

In summary, we showed that Duxap 8 promoted cell proliferation and inhibited cell apoptosis in colorectal cancer via targeting miR-519b-3p/ZNF277 axis. These findings suggest that Duxap 8 might serve as a promising molecular therapeutic target for the colorectal cancer.

\section{Acknowledgments}

These authors have contributed equally to this work: Hailiang Liang, Jin Wang, and Peng Zhang. These authors have contributed equally to this work: Wei Yang, Yang Yang, and Yin Zhi. Xiaoqiang Dong is corresponding author, and Wei $\mathrm{Wu}$ is co-corresponding author.

\section{Disclosure}

The authors report no conflicts of interest for this work.

\section{References}

1. Torre LA, Bray F, Siegel RL, Ferlay J, Lortet-Tieulent J, Jemal A. Global cancer statistics, 2012. CA Cancer J Clin. 2015;65:87-108.

2. Sadanandam A, Lyssiotis CA, Homicsko K, et al. A colorectal cancer classification system that associates cellular phenotype and responses to therapy. Nat Med. 2013;19:619.

3. Nishihara R, Wu K, Lochhead P, et al. Long-term colorectal-Cancer incidence and mortality after lower endoscopy. $N$ Engl $\mathrm{J}$ Med. 2013;369:1095-1105.

4. Damas ND, Marcatti M, Côme C, et al. SNHG5 promotes colorectal cancer cell survival by counteracting STAU1-mediated MRNA destabilization. Nat Commun. 2016;7:13875.

5. Han P, Li JW, Zhang BM, Lv JC, Cui BB. The lncRNA CRNDE promotes colorectal cancer cell proliferation and chemoresistance via miR-181a-5p-mediated regulation of $\mathrm{Wnt} / \beta$-catenin signaling. Mol Cancer. 2017;16:1.

6. Ding D, Li C, Zhao T, Li D, Zhang B. LncRNA H19/miR-29b-3p/PGRN axis promoted epithelial-mesenchymal transition of colorectal cancer cells by acting on Wnt signaling. Mol Cells. 2018;41(5):423-435.

7. Xu M, Chen X, Lin K, et al. The long noncoding RNA SNHG1 regulates colorectal cancer cell growth through interactions with EZH2 and miR-154-5p. Mol Cancer. 2018;17(1):141.

8. Bian Z, Jin L, Zhang J, et al. LncRNA-UCA1 enhances cell proliferation and 5-fluorouracil resistance in colorectal cancer by inhibiting miR-204-5p. Sci Rep. 2016;6:23892.
9. Dong-Liang C, Yun-Xin L, Jia-Xing Z, et al. Long non-coding RNA UICLM promotes colorectal cancer liver metastasis by acting as a ceRNA for microRNA-215 to regulate zeb2 expression. Theranostics. 2017;7(19):4836-4849.

10. Chen DL, Chen LZ, Lu YX, et al. Long noncoding RNA XIST expedites metastasis and modulates epithelial-mesenchymal transition in colorectal cancer. Cell Death Dis. 2017;8(8):e3011.

11. Sun M, Nie FQ, Zang C, et al. The pseudogene DUXAP8 promotes non-small-cell lung cancer cell proliferation and invasion by epigenetically silencing EGR1 and RHOB. Mol Ther. 2017;25(3):739-751.

12. Ma HW, Xie M, Sun M, Chen TY, Zhang ZH. The pseudogene derived long noncoding RNA DUXAP8 promotes gastric cancer cell proliferation and migration via epigenetically silencing PLEKHO1 expression. Oncotarget. 2014;8(32):52211-52224.

13. Lian Y, Yang J, Lian Y, Xiao $\mathrm{C}, \mathrm{Hu} \mathrm{X}, \mathrm{Xu}$ H. DUXAP8, a pseudogene derived lncRNA, promotes growth of pancreatic carcinoma cells by epigenetically silencing CDKN1A and KLF2. Cancer Commun (Lond). 2018;38(1):64.

14. Wang H, Huo X, Yang XR, et al. STAT3-mediated upregulation of IncRNA HOXD-AS1 as a ceRNA facilitates liver cancer metastasis by regulating SOX4. Mol Cancer. 2017;16(1):136. doi:10.1186/ s12943-017-0680-1

15. Lin MG, Hong YK, Zhang Y, Lin BB, He XJ. Mechanism of lncRNA DUXAP8 in promoting proliferation of bladder cancer cells by regulating PTEN. Eur Rev Med Pharmacol Sci. 2018;22(11):3370-3377.

16. $\mathrm{Xu} \mathrm{LJ}, \mathrm{Yu} \mathrm{XJ}$, Wei B, et al. Long non-coding RNA DUXAP8 regulates proliferation and invasion of esophageal squamous cell cancer. Eur Rev Med Pharmacol Sci. 2018;22(9):2646-2652.

17. Huang T, Wang X, Yang X, et al. Long non-coding RNA DUXAP8 enhances renal cell carcinoma progression via downregulating miR-126. Med Sci Monit. 2018;24:7340-7347.

18. Jiang H, Shi X, Ye G, et al. Up-regulated long non-coding RNA DUXAP8 promotes cell growth through repressing Krüppel-like factor 2 expression in human hepatocellular carcinoma. Onco Targets Ther. 2019;12:7429-7436.

19. Cesana M, Cacchiarelli D, Legnini I, et al. A long noncoding RNA controls muscle differentiation by functioning as a competing endogenous RNA. Cell. 2011;147(2):358-369. doi:10.1016/j.cell.2011. 09.028

20. Luo J, Liu L, Zhou N, Shen J, Chen M. MiR-519b-3p promotes responsiveness to preoperative chemoradiotherapy in rectal cancer patients by targeting ARID4B. Gene. 2018;655:84-90.

21. Shen Z, Zhan G, Deng H, Kang C, Guo J. [Growth inhibitory effect of microrna-519b-3p on larynx squamous hep-2 cells]. Zhonghuaer Bi Yan Hou Tou Jing Wai Ke Za Zhi. 2014;49(2):151-156. [Portuguese]

22. Zhang Y, Sun M, Chen Y, Li B. MiR-519b-3p inhibits the proliferation and invasion in colorectal cancer via modulating the uMtCK/ Wnt signaling pathway. Front Pharmacol. 2019;10:741. doi:10.3389/ fphar.2019.00741
OncoTargets and Therapy

\section{Publish your work in this journal}

OncoTargets and Therapy is an international, peer-reviewed, open access journal focusing on the pathological basis of all cancers, potential targets for therapy and treatment protocols employed to improve the management of cancer patients. The journal also focuses on the impact of management programs and new therapeutic
Dovepress

agents and protocols on patient perspectives such as quality of life, adherence and satisfaction. The manuscript management system is completely online and includes a very quick and fair peer-review system, which is all easy to use. Visit http://www.dovepress.com/ testimonials.php to read real quotes from published authors. 